\title{
Multiscale Active Contours
}

\author{
Xavier Bresson, Pierre Vandergheynst and Jean-Philippe Thiran \\ Signal Processing Institute (ITS), \\ Swiss Federal Institute of Technology (EPFL) \\ CH-1015 Lausanne, Switzerland, \\ \{Xavier.Bresson, Pierre.Vandergheynst, JP.Thiran\}@epfl.ch
}

\begin{abstract}
In this paper, we propose an evolution equation for the active contours in scale spaces. This evolution equation is based on the Polyakov functional that has been first introduced in physics and has been then used in image processing in [17] for image denoising. Our active contours are hypersurfaces implicitly and intrinsically represented by a level set function embedded in a scale space. The scale spaces used in our approach are defined by a family of metric tensors given by the general heat diffusion equation. The well-known scale spaces such as the linear scale space, i.e. the Gaussian scale space, the Perona-Malik scale space, the mean curvature scale space and the total variation scale space can be used in this framework. A possible application of this technique is in shape analysis. For example, our multiscale segmentation technique can be coupled with the shape recognition and the shape registration algorithms to improve their robustness and their performance.
\end{abstract}

\section{Introduction and Motivation}

This paper aims at introducing the scale parameter in the active contour formalism $[8,3,9]$ to define an object multiscale segmentation model. One of the main motivations to develop such a technique is to deal with the shape of objects at different scales of observation/resolution. Indeed, the works of Witkin [18] and Koenderink [10] have shown that the shape of objects changes according to the scale of observation used. At large scales, the global shape of the object can be observed since smaller shape features are suppressed. And at lower scales, finer characteristics appear in the shape of the object.

As a result, it appears natural to analyze a given image not only at one scale but at several scales of observation simultaneously. This will improve the robustness of classical image analysis techniques such as the shape recognition and the shape registration methods. For instance, it could be interesting to merge our multiscale segmentation algorithm with a multiscale shape model such as the one developed by Pizer et al. in [14] to create a multiscale recognition method. In [16], Schnabel and Arridge have proposed a method to extract the shape of objects at different levels of scale. They have then used the extracted multiscale 
shapes to localize and characterize shape changes at different levels of scale. They have applied their model to segment 3-D brain magnetic resonance images in order to quantify the structural deformations for patients having epilepsy. However, they have not taken into account the interdependance between space and scale in their segmentation model.

Any image can be observed at different scales thanks to a multi-resolution image representation called scale space by Witkin in [18] (see also the pioneering work of Iijima [7]). A scale space is a hierarchical decomposition of an image according to the scale of observation. It can also be seen as a family of gradually smoother versions of the original image.

The segmentation method we use in our work is the active contour model introduced by Kass et al. in [8]. We want to use this segmentation model to extract objects in scale spaces. For this purpose, we need to define an evolution equation for active contours propagating in scale spaces. Two main questions arise when we try to devise such an equation. How can the active contours be introduced into scale spaces and which scale spaces can be used? An answer to the first question is given by the Polyakov action that we will present in the next section. For the second question, we will use the family of scale spaces proposed by Eberly in [5] which includes the linear scale space, the Perona-Malik scale space, the curvature scale spaces and the total variation scale space.

\section{Polyakov Action}

The Polyakov action has been introduced in image processing by Sochen et al. in [17]. The Polyakov action is a functional that measures the weight of a map $X$ between the image manifold $\Sigma$ and the embedding manifold $M$ (see Figure 1). It is defined as follows:

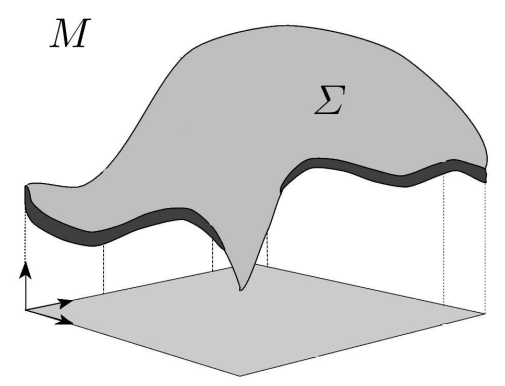

Fig. 1. The manifold $\Sigma$ embedded in $M$, reproduced from [17].

$$
P(X, \Sigma, M)=\int d^{m} \sigma g^{1 / 2} g^{\mu \nu} \partial_{\mu} X^{i} \partial_{\nu} X^{j} h_{i j}
$$


where $m$ is the dimension of $\Sigma, p$ the dimension of $M, g_{\mu \nu}$ and $h_{i j}$ are the metric tensors of manifolds $\Sigma$ and $M, g^{\mu \nu}$ is the inverse metric of $g_{\mu \nu}, g$ is the determinant of $g_{\mu \nu}, \mu, \nu=1, \ldots, m, i, j=1, \ldots, p, \partial_{\mu} X^{i}=\partial X^{i} / \partial \sigma^{\mu}$. Moreover, when identical indices appear one up and one down, they are summed over according to the Einstein summation convention.

If the Polyakov functional is minimized w.r.t. an embedding coordinate $X^{l}, g_{\mu \nu}$ and $h_{i j}$ being fixed, we get the following flow acting on $X^{l}$ :

$$
\partial_{t} X^{l}=g^{-1 / 2} \partial_{\mu}\left(g^{1 / 2} g^{\mu \nu} \partial_{\nu} X^{l}\right)+\Gamma_{j k}^{l} \partial_{\mu} X^{j} \partial_{\nu} X^{k} g^{\mu \nu} \quad \text { for } 1 \leq l \leq p,
$$

where $\Gamma_{j k}^{l}$ is the Levi-Civita connection.

Sochen et al. have proved in [17] that different choices of the metric tensor $g_{\mu \nu}$ in Equation (2) give the most well-known scale spaces: the linear scale space, the scale space of Perona-Malik, the mean curvature scale space and the total variation scale space. They have also proposed a new scale space to enhance image quality. They have called it the Beltrami flow since they have used the Beltrami operator which generalizes the Laplace operator in non-flat manifolds.

\section{Active Contours in Scale Spaces}

\subsection{Active Contours in Euclidean Spaces}

Following the first model of active contours proposed by Kass et al. in [8], Caselles et al. in [3] and Kichenassamy et al. in [9] have proposed an energy functional invariant w.r.t. the curve parametrization:

$$
F^{g a c}(C)=\oint_{0}^{L(C)} f d s,
$$

where $d s$ is the Euclidean element of length, $L(C)$ is the length of the curve $C$ and $f$ is an edge detecting function that vanishes at object boundaries. The calculus of variations provides the Euler-Lagrange equation of the functional $F^{g a c}$ and the gradient descent method gives the flow that minimizes $F^{g a c}$ :

$$
\partial_{t} C=(\kappa f-\langle\nabla f, \mathcal{N}\rangle) \mathcal{N}
$$

where $\kappa$ is the curvature and $\mathcal{N}$ the normal to the curve. Osher and Sethian have introduced in [12] the implicit and intrinsic level set representation of contours to efficiently solve the contour propogation problem and to deal with topological changes. The equation (4) can be written in the level set form:

$$
\partial_{t} \phi=\left(\kappa f+\left\langle\nabla f, \frac{\nabla \phi}{|\nabla \phi|}\right\rangle\right)|\nabla \phi|
$$

where $\phi$ is the level set function embedding the active contour $C$. 


\subsection{Scale Spaces}

In the previous section, the active contours have been defined in the Euclidean space. We want to put them in scale spaces by changing the embedding Euclidean manifold into the scale spaces. The question is which scale spaces will we use. In paper [5], Eberly has studied the geometry of a large class of scale spaces and he has defined for them the general metric tensor:

$$
\left[h_{i j}\right]=\operatorname{diag}\left(\frac{1}{c^{2}} I_{n}, \frac{1}{c^{2} \rho^{2}}\right),
$$

where $n$ is the spatial dimension, $c$ and $\rho$ are two functions that physically correspond to the conductance and the density functions in the general model of heat diffusion transfert.

Eberly has also defined in [5] the natural diffusion equation in any scale space as follows: the left-hand side of the diffusion equation is given by one application of the scale derivative and the right-hand side by two applications of the spatial derivative. The natural diffusion equation in a scale space is therefore

$$
\nabla_{\sigma}^{s s} u=\left(\nabla_{x_{1}, \ldots, x_{n}}^{s s}\right)^{2} u,
$$

where $\nabla_{\sigma}^{s s}$ is the scale derivative operator and $\nabla_{x_{1} \ldots \ldots x_{n}}^{s s}$ the spatial derivative operator. These operators are determined using the tensor metric (6) and the following differential geometry formulae:

$$
\begin{aligned}
\nabla_{x_{1}, \ldots, x_{n}}^{s s} & =\left(\begin{array}{c}
\sqrt{h^{x_{1} x_{1}}} \partial_{x_{1}} \\
\vdots \\
\sqrt{h^{x_{n} x_{n}}} \partial_{x_{n}}
\end{array}\right)=c \nabla, \\
\nabla_{\sigma}^{s s} & =\sqrt{h^{\sigma \sigma}} \partial_{\sigma}=c \rho \partial_{\sigma} .
\end{aligned}
$$

Hence, the diffusion equation (7) is equal to

$$
\partial_{\sigma} u=\frac{1}{\rho} \nabla \cdot(c \nabla u),
$$

which corresponds to the general heat diffusion equation with the conductance function $c$ and the density function $\rho$. The choice of the functions $c$ and $\rho$ determines the scale space and the diffusion equation we use. For examples,

$$
\left\{\begin{array}{lll}
\text { for } c=\sigma, \rho=1 & u_{\sigma}=\sigma \Delta u & \text { Linear Scale Space, } \\
\text { for } c=\exp \left(-\alpha|\nabla u|^{2}\right), \rho=1 & u_{\sigma}=\nabla \cdot(c \nabla u) & \text { Perona-Malik Flow, } \\
\text { for } c=\rho=\frac{1}{|\nabla u|} & u_{\sigma}=\nabla \cdot\left(\frac{\nabla u}{|\nabla u|}\right)|\nabla u| & \text { Mean Curvature Flow, } \\
\text { for } c=\frac{1}{|\nabla u|}, \rho=1 & u_{\sigma}=\nabla \cdot\left(\frac{\nabla u}{|\nabla u|}\right) & \text { Total Variation Flow, }
\end{array}\right.
$$

Eberly has proved in [5] that the linear scale space is hyperbolic and translation, rotation and scale invariant. The second scale space is given by the non-linear anisotropic diffusion equation of Perona and Malik proposed in [13]. The third one is the mean curvature flow introduced in the level set framework by Osher and Sethian in [12] and the fourth scale space is produced by the total variation flow defined by Rudin, Osher and Fatemi in [15]. 


\subsection{General Evolution Equation for Active Contours in Scale Spaces}

In the context of the Polyakov action, we look for the map $X$, the tensor $g_{\mu \nu}$ of the active contour manifold and the tensor $h_{i j}$ of the embedding space that lead to an evolution equation for active contours in the scale spaces. We choose the map $X$ as $X:\left(x_{1}, \ldots, x_{n}, \sigma\right) \rightarrow\left(x_{1}, \ldots, x_{n}, \sigma, \phi\right)$, where $\phi$ is the level set function representing the active contour. The metric tensor $h_{i j}$ of the embedding space is given by the Equation (6). The last choice concerns the tensor $g_{\mu \nu}$ of the active contour manifold. We choose the induced metric tensor on $\Sigma[17]$ :

$$
g_{\mu \nu}=\partial_{\mu} X^{i} \partial_{\nu} X^{j} h_{i j} .
$$

This choice is motivated by the classical works concerning the active contours [3, 9,4]. The Polyakov functional corresponding to the induced metric is the Euler functional:

$$
S(X)=\int d^{m} \sigma g^{1 / 2},
$$

which defines the (hyper-)aera of the (hyper-)surface $\Sigma$. The maps $X$ that minimize $S$ for any manifold $\Sigma$ embedded in any manifold $M$ are called harmonic maps. Harmonic maps are the generalization of geodesics and minimal surfaces (see $[3,9,4])$ to higher dimensional manifolds and for higher embedding manifolds.

The minimization of $S$ w.r.t. the component $X^{l}$ gives the generalized mean curvature flow, see [17], in any embedding manifold $M$ defined by the metric $h_{i j}$ :

$$
\partial_{t} X^{l}=\underbrace{g^{-1 / 2} \partial_{\mu}\left(g^{1 / 2} g^{\mu \nu} \partial_{\nu} X^{l}\right)}_{(13.1)}+\Gamma_{j k}^{l} \partial_{\mu} X^{j} \partial_{\nu} X^{k} g^{\mu \nu} \equiv \mathbf{H}^{l}=g^{-1 / 2} \mathbf{K}^{l}
$$

whose term (13.1) is the Laplace-Beltrami operator and $\mathbf{H}$ is the mean curvature vector generalized to any manifold $\left(M, h_{i j}\right)$.

Proposition 1: As in [3, 9, 4], we introduce a weighting function $f$ in the Euler fonctional:

$$
S_{f}(X)=\int d^{m} \sigma f(X) g^{1 / 2}
$$

The evolution equation minimizing this functional w.r.t. the l-th component of $X$ is [2]:

$$
\partial_{t} X^{l}=f \mathbf{H}^{l}+\partial_{k} f g^{\mu \nu} \partial_{\mu} X^{k} \partial_{\nu} X^{l}-\frac{m}{2} \partial_{k} f h^{k l}
$$

Application 1: The geodesic/geometric active contour evolving in the 2-D Euclidean space proposed in $[3,9]$ can be recovered. Indeed, if we take $X \equiv C$ and 
$h_{i j}=\delta_{i j}$, the evolution equation defined in Equation (15) becomes (up to a constant) the well-known flow

$$
\partial_{t} C=f \kappa \mathcal{N}-\frac{1}{2}\langle\nabla f, \mathcal{N}\rangle \mathcal{N}
$$

Application 2: The evolution equation of the level set function embedding the active contour can also be revisited. If we choose $X \equiv S:(x, y) \rightarrow(x, y, \phi(x, y))$, $h_{i j}=\delta_{i j}$, the energy is $E_{e s}=\int f \sqrt{1+|\nabla \phi|^{2}} d x d y$ and the flow acting on the level set component $\phi$ is:

$$
\partial_{t} \phi=f H_{e s}+g_{e s}^{-1 / 2}\langle\nabla f, \nabla \phi\rangle=g_{e s}^{-1 / 2}\left(f K_{e s}+\langle\nabla f, \nabla \phi\rangle\right)=g_{e s}^{-1 / 2} F_{e s}
$$

where $g_{e s}=1+|\nabla \phi|^{2}$ and $K_{e s}$ is the Euclidean mean curvature of the surface $X$ such that $K_{e s}=\frac{\left(1+\phi_{x}^{2}\right) \phi_{y y}-2 \phi_{x} \phi_{y} \phi_{x y}+\left(1+\phi_{y}^{2}\right) \phi_{x x}}{g_{e s}^{3 / 2}}$ (see [17]). The equation of the level set function $\phi_{t}=g_{e s}^{-1 / 2} F_{e s}$ implies that the surface $S$ evolves according to $S_{t}=F_{e s} \mathcal{N}_{S}$ where $\mathcal{N}_{S}=g_{e s}^{-1 / 2}\left(-\phi_{x},-\phi_{y}, 1\right)$. This means that the level sets of $\phi$ move according to the equation:

$$
\begin{aligned}
C_{t} & =P_{z} S_{t}=g_{e s}^{-1 / 2}|\nabla \phi| F_{e s} \mathcal{N}_{C}=r(\phi) F_{e s} \mathcal{N}_{C} \\
& =\left(f K_{e s} r(\phi)-\left\langle\nabla f, \mathcal{N}_{C}\right\rangle r^{2}(\phi)\right) \mathcal{N}_{C}
\end{aligned}
$$

where $P_{z}$ is a projector onto the plane normal to the $\phi$-axis, $\mathcal{N}_{C}=-\nabla \phi /|\nabla \phi|$ is the unit normal to the level sets and $r(\phi)=g_{e s}^{-1 / 2}|\nabla \phi|$. The equation (19) is close to the evolution equation (4) up to the surface mean curvature $K_{e s}$ and the function $r$. Function $r$ can be interpretated as an indicator of the height variation on the surface $S$ (see [1]). Indeed, $g_{e s}^{-1 / 2}$ is the ratio between the area of an infinitesimal surface in the domain $(x, y)$ and the corresponding area on the surface $S$. For flat surfaces, $r$ is equal to 0 and it is close 1 near edges. Finally the function $r$ is constant a.e. when $\phi$ is a signed distance function.

We propose the following evolution equation for active contours in the scale spaces.

Proposition 2: Given the induced metric tensor, Equation (11), the harmonic map $X$ defined by $\left(x_{1}, \ldots, x_{n}, \sigma\right) \rightarrow\left(x_{1}, \ldots, x_{n}, \sigma, \phi\right)$ and the weighting function $f=f\left(x_{1}, \ldots, x_{n}, \sigma\right)$, the evolution equation of the $(n+2)$-th component of $X$, i.e. the level set component $\phi$, is equal, according to (15), to:

$$
\partial_{t} \phi=f H_{s s}+\left\langle\nabla^{\star} f, \nabla^{\star} \phi\right\rangle_{\left(g^{\mu \nu}\right)},
$$

whose $H_{s s}=g^{-1 / 2} K_{s s}=\mathbf{H}^{n+2}$ is the $(n+2)$-th component of the mean curvature vector (13) generalized to scale spaces, $\nabla^{\star}=\left(\nabla, \partial_{\sigma}\right)$ and $\langle., .\rangle_{\left(g^{\mu \nu}\right)}$ is the inner product w.r.t. the metric $g^{\mu \nu}$ such that

$$
\left\langle V_{1}, V_{2}\right\rangle_{\left(g^{\mu \nu}\right)}=V_{1}^{t}\left(g^{\mu \nu}\right) V_{2}=V_{1 \mu} g^{\mu \nu} V_{2 \nu}
$$


where $g=1+|\nabla \phi|^{2}+\rho^{2} \phi_{\sigma}^{2}$. The energy of the multiscale active contour is computed according to Equation (14):

$$
E_{s s}=\int f \sqrt{1+|\nabla \phi|^{2}+\rho^{2} \phi_{\sigma}^{2}} \underbrace{\prod_{1 \leq i \leq n} \frac{d x_{i}}{c} \frac{d \sigma}{c \rho}}_{(22.1)},
$$

whose term (22.1) is the infinitesimal volume in the scale spaces defined by the metric tensor (6).

The evolution equation of the level set function $\phi$ is:

$$
\partial_{t} \phi=g^{-1 / 2} f K_{s s}+\left\langle\nabla^{\star} f, \nabla^{\star} \phi\right\rangle_{\left(g^{\mu \nu}\right)}=g_{e s}^{-1 / 2} F_{s s},
$$

where $g_{e s}=1+\left|\nabla^{\star} \phi\right|^{2}$. Hence the surface $S$ evolves according to $S_{t}=F_{s s} \mathcal{N}_{S}$ and the level sets of $\phi$ move according to:

$$
C_{t}=P_{z} S_{t}=\left(f K_{s s} r_{2}(\phi, c, \rho)-\left\langle\nabla^{\diamond} f, \mathcal{N}_{C}\right\rangle r_{2}^{2}(\phi, c, \rho)\right) \mathcal{N}_{C},
$$

with the operator $\nabla^{\diamond}=\frac{1}{c^{2} \rho^{2}}\left(\nabla f, \rho^{2} \partial_{\sigma}\right), \mathcal{N}_{C}=-\nabla^{\star} \phi /\left|\nabla^{\star} \phi\right|$ and $r_{2}(\phi, c, \rho)=$ $\left|\nabla^{\star} \phi\right| / g^{1 / 2}$.

\subsection{Application to the Linear Scale Space}

The linear scale space is obtained when $c=\sigma$ and $\rho=1$. In this case, the energy of the multiscale active contour is for $n=2$ :

$$
E_{l s s}=\int f \sqrt{1+|\nabla \phi|^{2}+\rho^{2} \phi_{\sigma}^{2}} \frac{d x d y d \sigma}{\sigma^{3}},
$$

and the flow of $\phi$ (embedding the active contour) is:

$$
\partial_{t} \phi=f H_{l s s}+\frac{1}{g}\left\langle\nabla^{\star} f, \nabla^{\star} \phi\right\rangle_{l s s},
$$

where $g=\frac{1}{\sigma^{6}}\left(1+\phi_{x}^{2}+\phi_{y}^{2}+\phi_{\sigma}^{2}\right),\langle., .\rangle_{l s s}$ is the inner product in the linear scale space defined by $\left\langle V_{1}, V_{2}\right\rangle_{l s s}=\frac{1}{\sigma^{2}}\left\langle V_{1}, V_{2}\right\rangle$ and $H_{l s s}$ is the mean curvature in the linear scale space computed using Equation (13):

$$
H_{l s s}=\underbrace{\frac{1}{g_{e s}} \phi_{\mu \nu} g^{\mu \nu}}_{(27.1)}-\underbrace{2 \frac{\phi_{\mu}}{\sigma} g^{\mu \sigma}}_{(27.2)}
$$

where $g_{e s}=1+\phi_{x}^{2}+\phi_{y}^{2}+\phi_{\sigma}^{2}$ and the components of $g^{\mu \nu}$ are:

$$
\begin{aligned}
& g^{x x}=\frac{1}{\sigma_{1}^{4}}\left(1+\phi_{y}^{2}+\phi_{\sigma}^{2}\right), \quad g^{x y}=-\frac{1}{\sigma_{1}^{4}} \phi_{x} \phi_{y}, \\
& g^{y y}=\frac{1}{\sigma_{1}^{4}}\left(1+\phi_{x}^{2}+\phi_{\sigma}^{2}\right), \quad g^{x \sigma}=-\frac{1}{\sigma_{1}^{4}} \phi_{x} \phi_{\sigma}, \\
& g^{\sigma \sigma}=\frac{1}{\sigma^{4}}\left(1+\phi_{x}^{2}+\phi_{y}^{2}\right), \quad g^{y \sigma}=-\frac{1}{\sigma^{4}} \phi_{y} \phi_{\sigma} .
\end{aligned}
$$

Roughly speaking, the term (27.1) corresponds to the Euclidean part of the mean curvature and the second term (27.2) to the Riemannian part. 


\section{Multiscale Image Features}

\subsection{Multiscale Edges}

In the previous section, we have defined a multiscale segmentation model which can capture image features representing by the function $f$. There exists different local multiscale image features but we will use the most common one, the norm of the image gradient, which is equal to $\left|\nabla_{s s} f\right|=\left(c^{2} f_{x_{1}}^{2}+\ldots+c^{2} f_{x_{n}}^{2}+c^{2} \rho^{2} f_{\sigma}^{2}\right)^{1 / 2}$ in the scale spaces. After that, we will extract the ridges of $\left|\nabla_{s s} f\right|$, the multiscale gradient norm.

Ridges can be defined by different ways (see [6]). In our approach, we have used the definition developed in Section 2.3 of [6]. In this case, Morse describes in [11] ridges of a 2-D feature image $f$ as points which have local maximum in $f$ along the direction of the greatest concavity of $f$. Thus, at a ridge point the direction of greatest curvature of $f$ is the cross-ridge direction and the value of $f$ is greater than the neighboring points on either side of it. This definition can be extended to higher dimensions. Indeed, let us consider a function $f$ in an $n$-D space and let us denote $\lambda_{1}, \ldots, \lambda_{n}$ with $\left|\lambda_{1}\right| \leq \ldots \leq\left|\lambda_{n}\right|$ and $e_{1}, \ldots, e_{n}$ the eigenvalues and the corresponding eigenvectors of the $n \times n$ matrix of the second derivatives. A point in an $n$-D space is an $m$-D ridge $(m<n)$ in $f$ if for all $i<n-m$,

$$
\left\{\begin{array}{c}
\lambda_{i}<0 \\
e_{i} \cdot \nabla f=0
\end{array}\right.
$$

In the case of the linear scale space with $n=2$ spatial dimensions, the Hessian matrix is different to the Euclidean one. Using the tensor metric $h_{i j}$ defined in Equation (6), with $c=\sigma$ and $\rho=1$, the Hessian matrix that includes the interdependence of space and scale is given in [11] by:

$$
\nabla_{l s s}^{2}=\left[\begin{array}{ccc}
\sigma^{2} \frac{\partial^{2} f}{\partial x^{2}}-\sigma \frac{\partial f}{\partial \sigma} & \sigma^{2} \frac{\partial^{2} f}{\partial x \partial y} & \sigma \frac{\partial^{2} f}{\partial x \partial \sigma}+\sigma \frac{\partial f}{\partial x} \\
\sigma^{2} \frac{\partial^{2} f}{\partial x \partial y} & \sigma^{2} \frac{\partial^{2} f}{\partial y^{2}}-\sigma \frac{\partial f}{\partial \sigma} & \sigma \frac{\partial^{2} f}{\partial y \partial \sigma}+\sigma \frac{\partial f}{\partial y} \\
\sigma \frac{\partial^{2} f}{\partial x \partial \sigma}+\sigma \frac{\partial f}{\partial x} & \sigma \frac{\partial^{2} f}{\partial y \partial \sigma}+\sigma \frac{\partial f}{\partial y} & \sigma^{2} \frac{\partial^{2} f}{\partial \sigma^{2}}+\sigma \frac{\partial f}{\partial \sigma}
\end{array}\right] .
$$

As an example, let us consider the fractal image proposed by Von Koch (see the first row on Figure 2). The magnitude of the scale space gradient is $\left|\nabla_{l s s} I(x, y, \sigma)\right|=$ $\sigma \cdot\left(I_{x}^{2}+I_{y}^{2}+I_{\sigma}^{2}\right)^{1 / 2}$ (see the second row on Figure 2). And the ridges of the multiscale gradient norm are given in the third row on Figure 2.

\subsection{Gradient Vector Flow in Scale Spaces}

The gradient vector flow (GVF) has been introduced by $\mathrm{Xu}$ and Prince in [19]. The GVF field is a non-irrotational force field, namely $\mathbf{V}$, which can capture the object boundaries far from them and can deal with concave boundary regions. It is defined in a variational approach since the GVF field must minimize the 

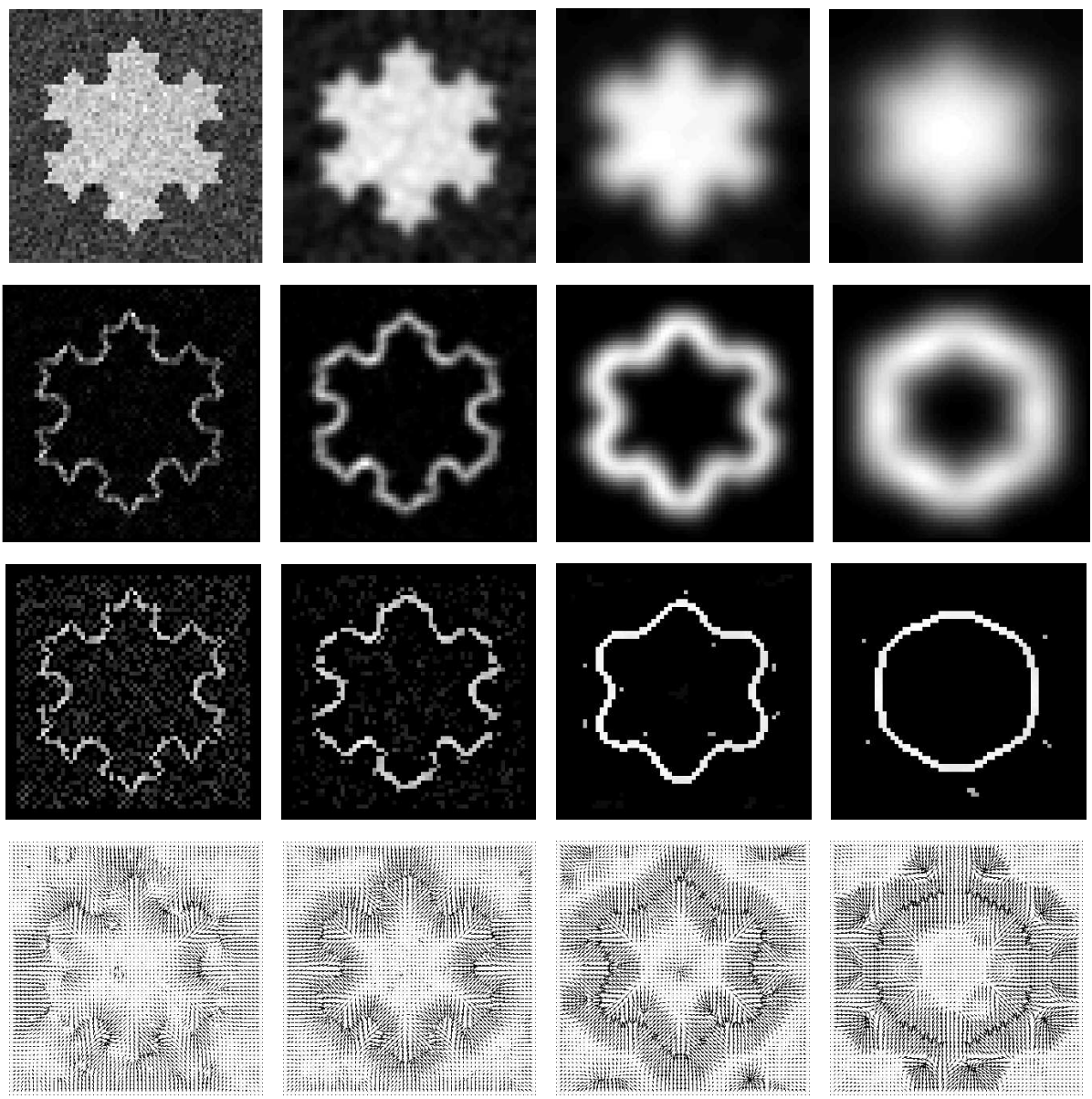

Fig. 2. First row: the Von Koch picture at four different scales of observation. The first image is the original image. Second row: the multiscale gradient of the Von Koch picture. Third row: the ridges of the multiscale gradient. Fourth row: GVFs of the ridge images.

following energy functional in the $n$-D Euclidean space:

$$
F^{g v f}(\mathbf{V})=\int \mu \underbrace{\sum_{i=1}^{n}\left(\left|\nabla V_{i}\right|^{2}\right)}_{(31.1)}+\underbrace{|\nabla f|^{2}|\mathbf{V}-\nabla f|^{2}}_{(31.2)} d \Omega
$$

where $V_{i}$ is the $i$-th component of the GVF field and $\mu$ is a constant which balances the contributions between the regularization term (31.1) and the data fidelity term (31.2). The minimization of the energy functional (31) is done using 
the calculus of variations and the gradient descent method which provide $n$ flows, one per component of the GVF field.

In this section we propose to extend the GVF to the scale spaces defined by the metric tensor (6). We realize this extension by simply changing the Euclidean terms by their Riemann equivalents. Thus, we replace the Euclidean gradient $\nabla$ by the scale space gradient $\nabla_{s s}$ and the Euclidean infinitesimal volume element $d \Omega$ by the scale space one $d \Omega_{s s}$, the energy (31) then becomes:

$$
F_{s s}^{g v f}(\mathbf{V})=\int \mu \sum_{i=1}^{n}\left(\left|\nabla_{s s} V_{i}\right|^{2}\right)+\left|\nabla_{s s} f\right|^{2}\left|\mathbf{V}-\nabla_{s s} f\right|^{2} d \Omega_{s s}
$$

The Frechet derivative of $F_{s s}^{g v f}$ w.r.t. $V_{i}$ in the $\xi$ direction is

$$
\begin{array}{r}
\left\langle\frac{\partial F_{s s}^{g v f}}{\partial V_{i}}, \xi\right\rangle=\int \xi \cdot\left[-\mu\left(\sum_{i=1}^{n} \partial_{x_{i}}\left(c^{2} \partial_{x_{i}} V_{i}\right)+\partial_{\sigma}\left(c^{2} \rho^{2} \partial_{\sigma} V_{i}\right)\right)+\right. \\
\left.\left|\nabla_{s s} f\right|^{2}\left|V_{i}-\left(\nabla_{s s} f\right)_{i}\right|^{2}\right] d \Omega_{s s} .
\end{array}
$$

Then, the flow minimizing $F_{s s}^{g v f}$ w.r.t. $V_{i}$ is

$$
\partial_{t} V_{i}=\mu\left(\sum_{i=1}^{n} \partial_{x_{i}}\left(c^{2} \partial_{x_{i}} V_{i}\right)+\partial_{\sigma}\left(c^{2} \rho^{2} \partial_{\sigma} V_{i}\right)\right)-\left|\nabla_{s s} f\right|^{2}\left|V_{i}-\left(\nabla_{s s} f\right)_{i}\right|^{2}
$$

For the linear scale space and $n=2$, the GVFs for $i=x, y, \sigma$ are:

$$
\partial_{t} V_{i}(x, y, \sigma)=\mu\left(\sigma^{2} \Delta V_{i}+2 \sigma \partial_{\sigma} V_{i}\right)-\sigma^{2}|\nabla f|^{2}\left(V_{i}-\sigma \partial_{i} f\right)
$$

Figure 2 (fourth row) presents the GVFs of the ridges images (third row).

\section{$5 \quad$ Result}

We have applied the evolution equation (26) in the linear scale space to segment the Von Koch picture at different scales of observation. The Figure 3 presents our multiscale snake evolving in the linear scale space at different times and the Figure 4 shows the segmentation process at four different scales.

\section{Conclusion}

In this paper, we have introduced the scale parameter in the active contour formalism by defining an evolution equation for the active contours in the scale spaces based on the general heat diffusion equation. We have supposed that the metric tensor $g_{\mu \nu}$ of the level set manifold is the induced metric tensor, i.e. the case where the active contours are harmonic maps. We could consider another choice for $g_{\mu \nu}$ such as a diagonal tensor (see [2]). Future works will be focused on integrating this multiscale segmentation technique into shape analysis methods such as the shape recognition and the shape registration methods to improve their performance. 


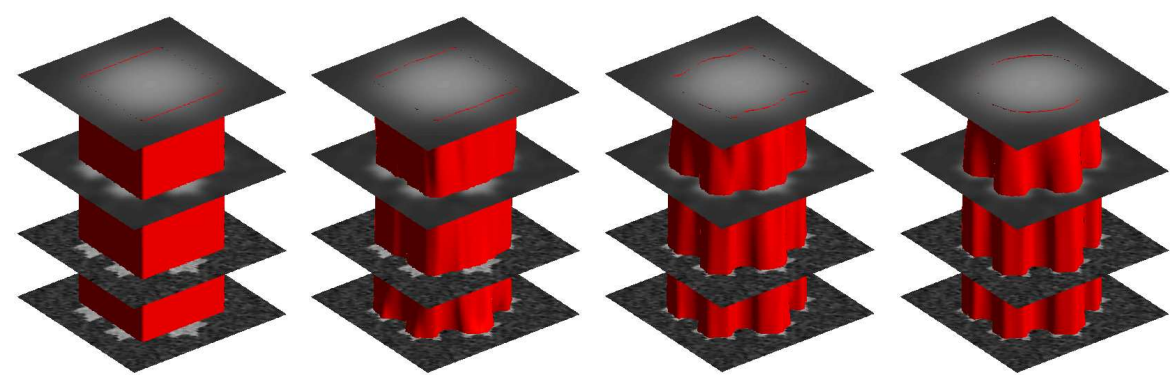

Fig. 3. Active contour evolving in the linear scale space.

\section{References}

1. G. Aubert and P. Kornprobst. Mathematical Problems in Image Processing, Partial Differential Equations and the Calculus of Variations. Springer, 2002.

2. X. Bresson, P. Vandergheynst, and J.-P-Thiran. Multiscale Active Contours - ITS Technical Report 12.04, 2004.

3. V. Caselles, R. Kimmel, and G. Sapiro. Geodesic Active Contours. International Journal of Computer Vision, 22(1):61-79, 1997.

4. V. Caselles, R. Kimmel, G. Sapiro, and C. Sbert. Minimal Surfaces: A Three Dimensional Segmentation Approach. IEEE Transactions on Pattern Analysis and Machine Intelligence, 19(4):394-398, 1997.

5. D. Eberly. A Differential Geometric Approach to Anisotropic Diffusion in Geometry-Driven Diffusion in Computer Vision. Computational Imaging and Vision, 1:371-392, 1994.

6. D.H. Eberly. Geometric Methods For Analysis Of Ridges In n-Dimensional Images - PhD Thesis - University of North Carolina, 1994.

7. S. Ishikawa J. Weickert and A. Imiya. Linear Scale-Space Has First Been Proposed In Japan. Mathematical Imaging and Vision, 10:237-252, 1999.

8. M. Kass, A. Witkin, and D. Terzopoulos. Snakes: Active Contour Models. International Journal of Computer Vision, pages 321-331, 1987.

9. S. Kichenassamy, A. Kumar, P. Olver, A. Tannenbaum, and A.J. Yezzi. Conformal Curvature Flows: From Phase Transitions to Active Vision. In Archive for Rational Mechanics and Analysis, volume 134, pages 275-301, 1996.

10. J. J. Koenderink. The Structure of Images. Biological Cybernetics, 50:363-370, 1984.

11. B.S. Morse. Computation Of Object Cores From Grey-Level Images - PhD Thesis - University of North Carolina, 1994.

12. S. Osher and J.A. Sethian. Fronts Propagating with Curvature-Dependent Speed: Algorithms Based on Hamilton-Jacobi Formulations. Journal of Computational Physics, 79(1)(12-49), 1988.

13. P. Perona and J. Malik. Scale-Space and Edge Detection Using Anisotropic Diffusion. IEEE Transactions on Pattern Analysis and Machine Intelligence, 1252(629639), 1990.

14. S.M. Pizer, D. Eberly, B.S. Morse, , and D. Fritsch. Zoom-Invariant Vision of Figural Shape: The Mathematics of Cores. Computer Vision and Image Understanding, 69:55-71, 1998. 

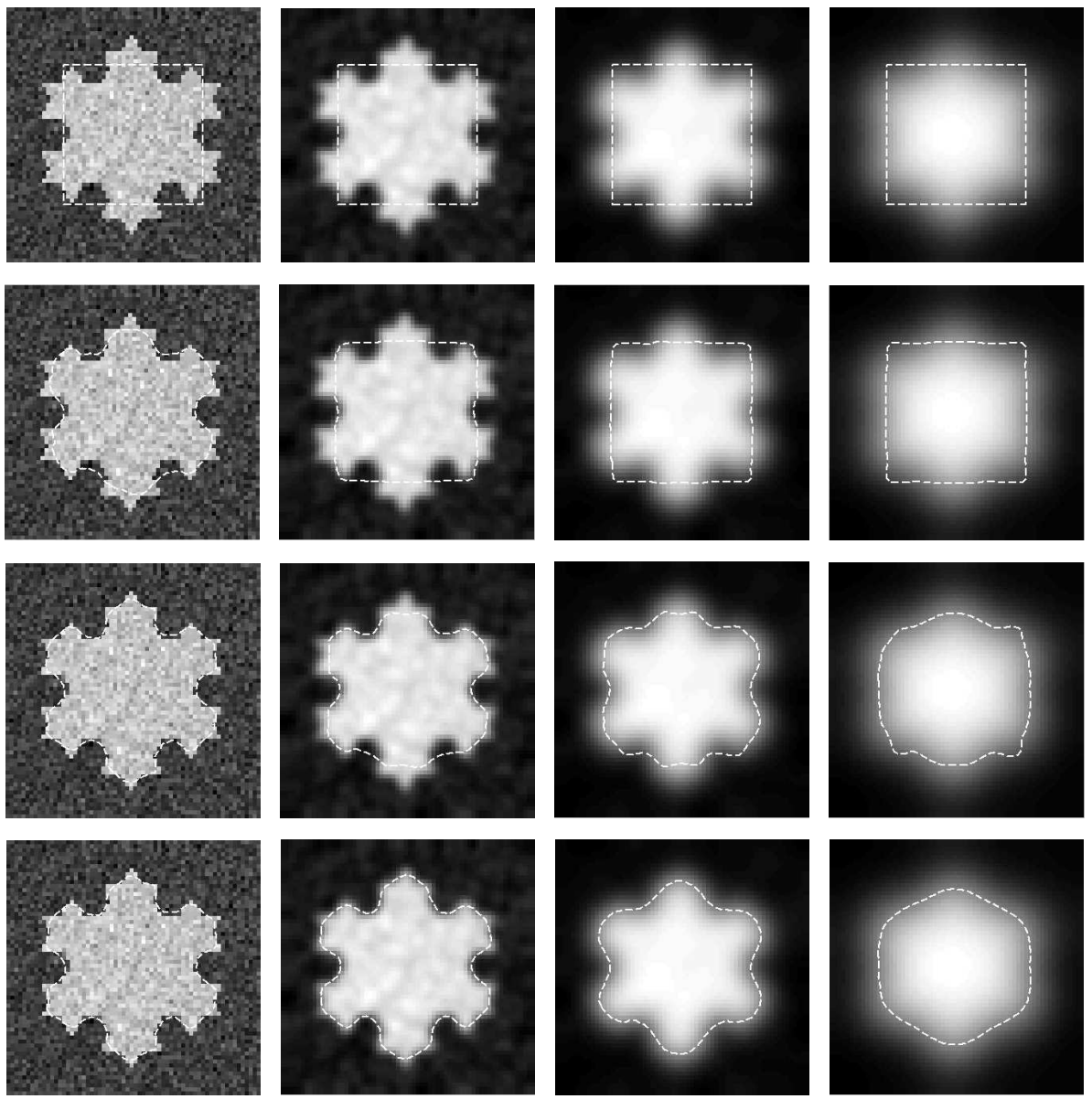

Fig. 4. Active contour evolving in the linear scale space at four different scales.

15. L. I. Rudin, S. Osher, and E. Fatemi. Nonlinear Total Variation Based Noise Removal Algorithms. Physica D, 60(1-4):259 - 268, 1992.

16. J. A. Schnabel and S.R. Arridge. Active Shape Focusing. Image and Vision Computing, 17(5-6):419-428, 1999.

17. N. Sochen, R. Kimmel, and R. Malladi. A General Framework For Low Level Vision. IEEE Transactions on Image Processing, 7(3):310 - 318, 1998.

18. A.P. Witkin. Scale-space filtering. In Proc. 8th International Joint Conference Artificial Intelligence, pages 1019-1022, 1983.

19. C. Xu and J. Prince. Snakes, shapes and gradient vector flow. IEEE Transaction on Image Processing, 7:359-369, 1998. 